\title{
History of the Mineral Sciences: Crystals and their Role in the Development of Atomic Theory

\author{
CHARLES A. GEIGER
}

Salzburg University

Presenting Author: ca.geiger@sbg.ac.at

In terms of the various natural science disciplines, the study of minerals is one of the oldest. In the Western World, major works on minerals were written in ancient Greece (e.g., Theophrastus, c.371-c.287 BC, "Peri Lithon") and Rome (e.g., "Pliny the Elder", AD 23-79, "Naturalis Historia"). Relatively little scientific advancement was made during the so-called "Dark Ages". A breakout was achieved in Europe in the late 1700s. Important discoveries were made and theories were proposed relating to crystallography and chemical crystallography. The "father of crystallography", René-Just Haüy, proposed a model (i.e., molécules intégrantes) to describe the microscopic nature of crystals starting around 1780. Various scientists such as Berzelius, Mitscherlich, Beudant, Wollaston and Kopp followed and researched crystal and elemental properties before 1850 . The disciplines of chemistry and mineralogy were closely interrelated. The discovery of isomorphism (solid solution) in crystals was an important step leading to the development of atomic theory. Terms such as atoms, molecules, corpuscles, and various other expressions for tiny particles were used interchangeably and a molecular approach, following Haüy, for interpreting microscopic crystal behavior was in fashion, but doubts existed. There was little physical understanding at the microscopic level and research focused mostly on determining the masses and charges of various elements.

The major breakthrough relating to crystals occurred with the discovery of X-ray diffraction in 1912 by von Laue and colleagues. It allowed the atomic structures of crystalline phases to be determined. At about the same time, the Rutherford-Bohr model (1913) for the atom was developed. The two Braggs, father and son, and their co-workers determined many mineral structures after 1912. However, a molecular approach for understanding silicates was still open into the 1920s. The "father of crystal chemistry" Goldschmidt made key contributions in the 1920s regarding atomic and ion radii and he expounded on solidsolution behavior. The importance of the sizes of ions was key in understanding many minerals, as was the nature of chemical bonding (e.g., Pauling - summarized in "The Nature of the Chemical Bond," 1939). "Modern mineralogy", a quantitative science, as it is largely understood today, emerged by roughly the mid 1930s. 